\title{
Shock interpretation and noninvasive hemodynamic monitoring with transthoracic echocardiography in an intensive care unit: a concise review
}

\author{
Kin-Bong Tang ${ }^{1} \rrbracket$, Chun-Wun Marten Lau ${ }^{2}$, Kin-Chio Li ${ }^{3} \rrbracket$ \\ Author affiliations: \\ 1. ORCID: 0000-0001-7801-4320; Department of Intensive Care, Pamela Youde Nethersole Eastern Hospital, 3 Lok Man \\ Road, Chai Wan, Hong Kong. \\ 2. ORCID: 0000-0001-8104-2322; Department of Intensive Care, Pamela Youde Nethersole Eastern Hospital, 3 Lok Man \\ Road, Chai Wan, Hong Kong. \\ 3. ORCID: 0000-0002-9528-2906; Department of Intensive Care, Pamela Youde Nethersole Eastern Hospital, 3 Lok Man \\ Road, Chai Wan, Hong Kong. \\ Correspondence: Dr. Chun-Wun Marten Lau; E-mail: wilson2606.mobile@gmail.com
}

\section{Abstract}

Circulatory failure and shock are common in critically ill patients, and the cause of shock is usually multifactorial. Transthoracic echocardiography is a noninvasive method to determine the contribution of various factors toward a patient's circulatory failure. Such factors include fluid status, cardiac contractility as well as vasomotor tone. Advances in echocardiographic measurements allow for the accurate estimation of a patient's loading status and cardiac contractility, which help to guide treatment strategy and monitor treatment response. This article offers an overview of echocardiographic and clinical parameters and aims to incorporate these findings into a methodical approach to the clinical management of shock.

Abbreviations: Ea - Arterial elastance; ECHO - Echocardiograph; Ed - Ventricular diastolic elastance; EDPVR - Enddiastolic pressure-volume relationship; Ees - Ventricular end-systolic elastance; EF - Ejection fraction; ESP - Endsystolic pressure; ESPVR - End-systolic pressure-volume relationship; GLS - Global longitudinal strain; ICU Intensive care unit; LA - Left atrium; LAP - Left atrial pressure; LV - Left ventricle; LVEDP - Left ventricular enddiastolic pressure; LVEDV - Left ventricular end-diastolic volume; LVESV - Left ventricular end-systolic volume; LVOT - Left ventricular outflow tract; LVOTacc - Left ventricular outflow tract mean flow acceleration; PAC - Pulmonary artery catheter; PCWP - Pulmonary capillary wedge pressure; PEEP - Positive end-expiratory pressure; PV loop Pressure-volume loop; PW - Pulse wave Doppler; RV - Right ventricle; RVSP - Right ventricular systolic pressure ; SV - Stroke volume; SVR - Systemic vascular resistance; TDI - Tissue Doppler imaging; TR - Tricuspid regurgitation; VA coupling - Ventriculo-arterial coupling; VTI - Velocity-time integral

Key words:_Transthoracic echocardiogram; Shock; Hemodynamic monitoring; ICU

Citation: Tang KB, Lau CWM, Li KC. Shock interpretation and noninvasive hemodynamic monitoring with transthoracic echocardiography in an intensive care unit: a concise review. Anaesth. pain intensive care 2021;25(6):819-827; DOI: 10.35975/apic.v25i6.1713

Received: October 5, 2021; Reviewed: October 10, 202; Accepted: October 25, 2021

\section{Introduction}

Shock is defined as a life-threatening circulatory failure associated with inadequate oxygen delivery to cells. ${ }^{1}$ Clinically, shock is classified according to its underlying forms, including cardiogenic shock, hypovolemic shock, distributive shock, and obstructive shock. A pulmonary artery catheter (PAC) was introduced in the 1970s for hemodynamic monitoring to provide data on the patients cardiac output and loading status. The use of PACs has decreased among ICUs worldwide since the 


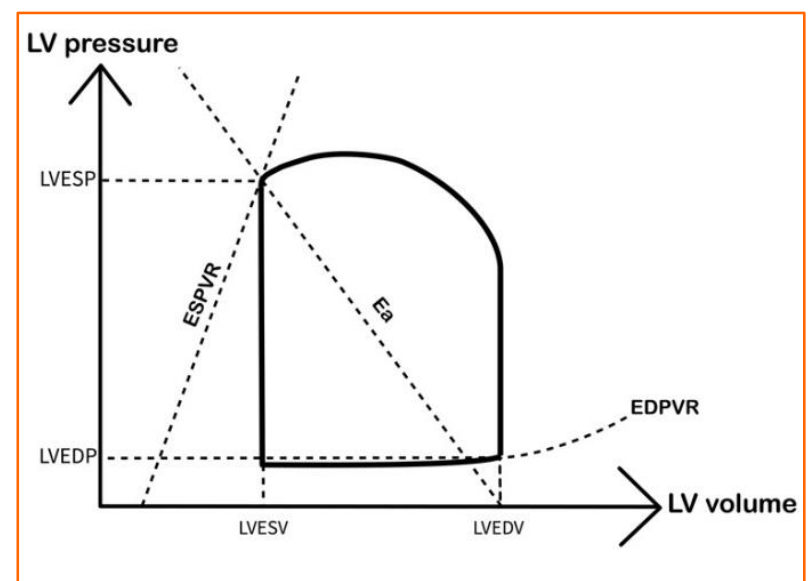

Figure 1: Pressure-volume loop of normal left ventricle. [LVESP, left ventricular end-systolic pressure; $L V E D P$, left ventricular end-diastolic pressure; LVESV, left ventricular end-systolic volume; LVEDV, left ventricular end-diastolic volume; EDPVR, end-diastolic pressurevolume relationship; ESPVR, end-systolic pressurevolume relationship; Ea, arterial elasticity. Stroke volume is LVEDV - LVESV].

year 2000, after the publication of several landmark studies demonstrating a lack of mortality benefit and the potential risk of catheter-related complications with PACs. ${ }^{2-6}$

Transthoracic echocardiography (ECHO) is an effective tool in a critical care setting, which enables us to assess the patients' cardiac loading status and the contractility. In contrast to ambulatory patients who undergo an elective echocardiographic study, critically ill patients are subject to minute-to-minute changes in physiological parameters due to the underlying pathology and treatment received. Various echocardiographic measuring tools have been developed to assess patients' cardiovascular parameters, which enable us to individualize the resuscitation strategy accordingly.anes ${ }^{7}$ In this review, we focus on the use of transthoracic echocardiograph-derived hemodynamic parameters in determining different components of shock and how those parameters could guide our management of shock.

\section{Pressure-volume loop}

The left ventricular pressure-volume loop (PV loop) (Figure 1) represents the relationship between pressure and volume within the ventricle during the cardiac cycle. Left ventricular preload is defined as the degree of tension on the ventricular wall muscle when it begins to contract, ${ }^{8}$ which is related to the initial myocardial fiber length prior to contraction. Left ventricular end-diastolic pressure (LVEDP) could serve as a surrogate of LV preload with respect to particular LV compliance.anes ${ }^{9}$ LV compliance reflects the change in LV filling pressure with respect to changes in LV volume, which relates to the likelihood of developing pulmonary edema with a further increase in preload with fluid challenge.anes ${ }^{9} \mathrm{On}$ the PV loop, passive ventricular filling occurs along with the left ventricular end-diastolic pressure-volume relationship (EDPVR), and the slope of the EDPVR is the reciprocal of ventricular compliance. The maximum pressure developed by the ventricle during contraction at any given volume is defined by the end-systolic pressure--volume relationship (ESPVR), representing the contractility of the left ventricle. Arterial elasticity (Ea) is defined as the change in arterial pressure for a given change in volume, which is calculated as left ventricular end systolic pressure (ESP) divided by stroke volume $(\mathrm{SV})(\mathrm{Ea}=\mathrm{ESP} / \mathrm{SV})$. Ea incorporates the principal elements of vascular load, and has been shown to be a valuable tool to assess arterial load and its impact on the left ventricle. ${ }^{10}$

\section{Transthoracic echocardio- gram parameters}

The vulnerability of the LV to an acute loading change is determined by ventricular stiffness and the end-systolic ventriculo-arterial interaction. These parameters can only be measured invasively by plotting the LV PV loop with different loading conditions, making it clinically inapplicable during resuscitation. Recent studies have introduced the concept of single beat-derived ventricular diastolic elastance (Ed), ventricular end-systolic elastance (Ees) and effective arterial elastance, ${ }^{10-13}$ which reflect the LV EDPVR, LV ESPVR and afterload relationship on the PV loop. These parameters are suggested to be load-independent and better reflect the contractility and compliance of the left ventricle.

\section{Left ventricular diastolic function and preload}

Left ventricular filling pressure refers to the pressure in the LV during the diastolic phase when it undergoes passive and active filling, which can be expressed as the mean pulmonary capillary wedge pressure (PCWP), mean left atrial pressure (LAP), and LV end-diastolic pressure (LVEDP). Mean LAP and mean PCWP are more clinically relevant definitions of LV filling pressure. They are better correlated with pulmonary capillary pressure, which is a primary determinant of fluid flux across the pulmonary capillary wall and the formation of lung edema ${ }^{14} \mathrm{An}$ algorithm was proposed in the guidelines for the evaluation of LV diastolic function of the American Society of Echocardiography to determine the mean LAP of patients with LV diastolic dysfunction based on mitral E/A ratio, peak E velocity, E/e' ratio, left atrial (LA) maximal volume index, and TR peak velocity. ${ }^{15}$ However, this guideline is based on 
expert consensus, and the applicability of this guideline to ICU patients is questionable.

Peak early mitral inflow velocity (mitral E wave) represents passive LV filling. Its peak velocity is directly related to LAP and inversely related to the time constant of LV relaxation. ${ }^{16}$ The velocity of mitral annular motion along the longitudinal plane during early diastole (mitral e' velocity) is measured using tissue Doppler imaging (TDI). By dividing E wave velocity by e' velocity to correct for the influence of relaxation, the E/e' ratio reflects a good correlation with LAP. Nagueh et al. ${ }^{17}$ demonstrated that $\mathrm{E} / \mathrm{e}^{\prime}$ is closely related to PCWP in patients in the ICU or Cath lab $(r=0.87)$. However, the effect of mechanical ventilation on transmitral flow and TDI should be taken into account. A randomized study on the impact of positive end-expiratory pressure (PEEP) on LV diastolic function measurement demonstrated that increasing PEEP from 0 to $12 \mathrm{cmH}_{2} \mathrm{O}$ will decrease transmitral $\mathrm{E}$ wave velocity and septal and lateral e', while E/A and E/e' will remain unaffected. ${ }^{18}$ The mitral E/e' ratio fails to correlate with PCWP in patients with an LV ejection fraction (EF) less than $30 \%,{ }^{19}$ thus limiting its use in cases of decompensated heart failure.

Diastole encompasses the time period during which the myocardium loses its ability to generate force and returns to an unstressed length and force. Left ventricular diastolic dysfunction refers to the situation where these processes are prolonged, slowed, or incomplete ${ }^{20}$ and hence the $\mathrm{LV}$ is unable to accommodate an adequate blood volume during diastole at volumes sufficient to maintain an appropriate SV. On the PV loop, LV diastolic function is reflected by the LVEDR. With increased LV chamber stiffness, the slope of the LVEDR curve is increased. The slope of the LVEDR curve can be calculated on ECHO by dividing LV pressure changes (E/e') by LV volume changes during filling (i.e., SV), which represent the LV operant Ed. ${ }^{11}$ The normal Ed value is $0.11 \pm 0.03$, and a study has shown that $\mathrm{Ed}$ increases with age and is higher among females than males. ${ }^{11}$

Ed is also significantly increased in patients with dilated cardiomyopathy compared to healthy individuals, whereas resting Ed independently correlates with exercise tolerance. ${ }^{21}$ However, no study has validated the use of Ed in patients on mechanical ventilation, nor has it been validated in critically ill patients on inotropic support. Further research is needed to investigate the applicability of Ed in the ICU setting.

\section{Left ventricular afterload}

Afterload is defined as the load against which the muscle exerts its contractile force and is determined by myocardial wall stress and input impedance. ${ }^{9}$ Afterload is determined by two components - the pulsatile component of central aortic mechanics and nonpulsatile peripheral vascular resistance. Systemic vascular resistance (SVR) only represents the nonpulsatile component. Arterial compliance reflects the pulsatile component, which is calculated by SV divided by pulse pressure. Effective Ea reflects the total afterload imposed on the LV and represents the complex association of different arterial properties, including wall stiffness, compliance and outflow resistance. ${ }^{22}$ Clinically, Ea can be defined as the capability of the arterial system to increase pressure when SV increases and can be estimated with end-systolic pressure (ESP) divided by $\mathrm{SV}(\mathrm{ESP} / \mathrm{SV}) .{ }^{10} \mathrm{ESP}$ can be derived from systolic blood pressure, as a study has shown that ESP is equal to $0.9 \mathrm{x}$ SBP. ${ }^{10}$ The normal value of $\mathrm{Ea}$ is $2.2 \pm 0.8 \mathrm{mmHg} / \mathrm{ml} .^{23}$ Studies have shown that Ea decreases during septic shock $\left.^{24}\right]$ and increases during heart failure. ${ }^{25}$

\section{Left ventricular systolic function and contractility}

The determination of left ventricular systolic function is one of the fundamental aims of echocardiograph assessment in the ICU. EF is frequently used to express LV contractility, which is the ratio of SV end-diastolic volume to LV end-diastolic volume (LVEDV). Both of these parameters are influenced by changes in preload and afterload; thus, LVEF is also load-dependent and cannot reflect LV contractility in altered loading status. Left ventricular global longitudinal strain (GLS) is another way to assess LV systolic function and contractility. However, GLS is also dependent on loading condition and affected by tachycardia. ${ }^{26,27} \mathrm{LV}$ ESPVR over a range of end-systolic points is relatively insensitive to cardiac loading and varies significantly in response to changes in $\mathrm{LV}$ contractility. LV Ees is the slope of LV ESPVR, which reflects changes in LV endsystolic volume (LVESV) and SV in response to LVESP changes. LV Ees is a reliable index of LV contractility, independent of loading status. Traditionally, Ees is invasively measured by plotting the pressure volume loop recording at different loading statuses, thus limiting its clinical application. Chen and coworkers described a single-beat method, with the use of ECHO, to measure Ees. ${ }^{12}$ This method is well validated against invasive assessment and is considered the gold standard for the estimation of Ees with ECHO. ${ }^{13}$ The acceleration of blood into the ascending aorta has been shown to be sensitive to inotropic states and relatively insensitive to the loading condition of the heart. ${ }^{28}$ Bauer and coworkers demonstrated a strong correlation between left ventricular outflow tract (LVOT) flow mean acceleration $($ LVOTacc $=$ LVOT Vmax/LVOT acceleration time $)$ and LV Ees $(r=0.85, p<0.001)$, which can be used as a surrogate for LV Ees. ${ }^{29}$ 
Accurate SV measurement is essential for the estimation of Ea and determination of the type of shock. SV is calculated by multiplying LVOT flow velocity-time integral (VTI) by LVOT area, the accuracy of which depends on the accurate measurement of LVOT VTI and LVOT diameter. LVOT flow velocity is recorded with pulsed-wave Doppler in either the apical 5-chamber view or apical long-axis view. The sample volume should be just proximal to the aortic valve, and the recording should show a smooth velocity curve with a well-defined peak and a narrow band of velocities throughout systole. When the sample volume is too close to the aortic valve, significant flow convergence will result in spectral dispersion. ${ }^{30}$ LVOT diameter is measured in the parasternal long-axis view during midsystole, from the inner edge to the inner edge of the septal endocardium and the anterior mitral leaflet, parallel to the aortic valve plane. ${ }^{30}$ LVOT diameter should be measured at the annular level or $2 \mathrm{~mm}$ below the annulus. A study has shown that, when compared to cardiac MRI measurements of stroke volume, using an LVOT diameter measured $5 \mathrm{~mm}$ and $10 \mathrm{~mm}$ below the edges of the annulus to calculate $\mathrm{SV}$ will result in significant underestimation. ${ }^{31}$

\section{ECHO parameter changes during shock}

Hypotension is a physiological state in which arterial blood pressure is abnormally low. When a reduction in blood pressure is severe enough to impair oxygen delivery to organs, resulting in cellular and organ dysfunction, then it is defined as shock. ${ }^{32}$ Since arterial blood pressure is a function of cardiac output and arterial load, ${ }^{33}$ alternation of these factors will result in hypotension. With ECHO measurements of the patient's myocardial contractility and loading status, the pathogenesis of shock can be clearly delineated to guide the treatment plan.

\subsection{Cardiogenic Shock}

An acute reduction in cardiac output due to impaired myocardial contractility will cause cardiogenic shock. On the PV loop, impaired contractility will result in flattening of the ESPVR curve with decreased Ees. ${ }^{34}$ Pathology causing impaired LV contractility may also affect LV compliance (e.g., myocardial infarction ${ }^{35}$ ] and stress cardiomyopathy, $\left.{ }^{36,37}\right]$ ), causing the LVEDR curve to shift upward as Ed increases. ${ }^{38}$ Hypotension triggers activation of the autonomic nervous system, resulting in vasoconstriction and increased arterial tone; therefore, Ea will increase. ${ }^{39}$ With the flattened ESPVR and increase in $\mathrm{Ea}, \mathrm{SV}$ will decrease ${ }^{40}$ ] and LVESV will increase, resulting in decreased LVEF. Blood will accumulate in the LV due to a decrease in SV, and the LVEDV will increase. ${ }^{41}$ In the case of chronic LV
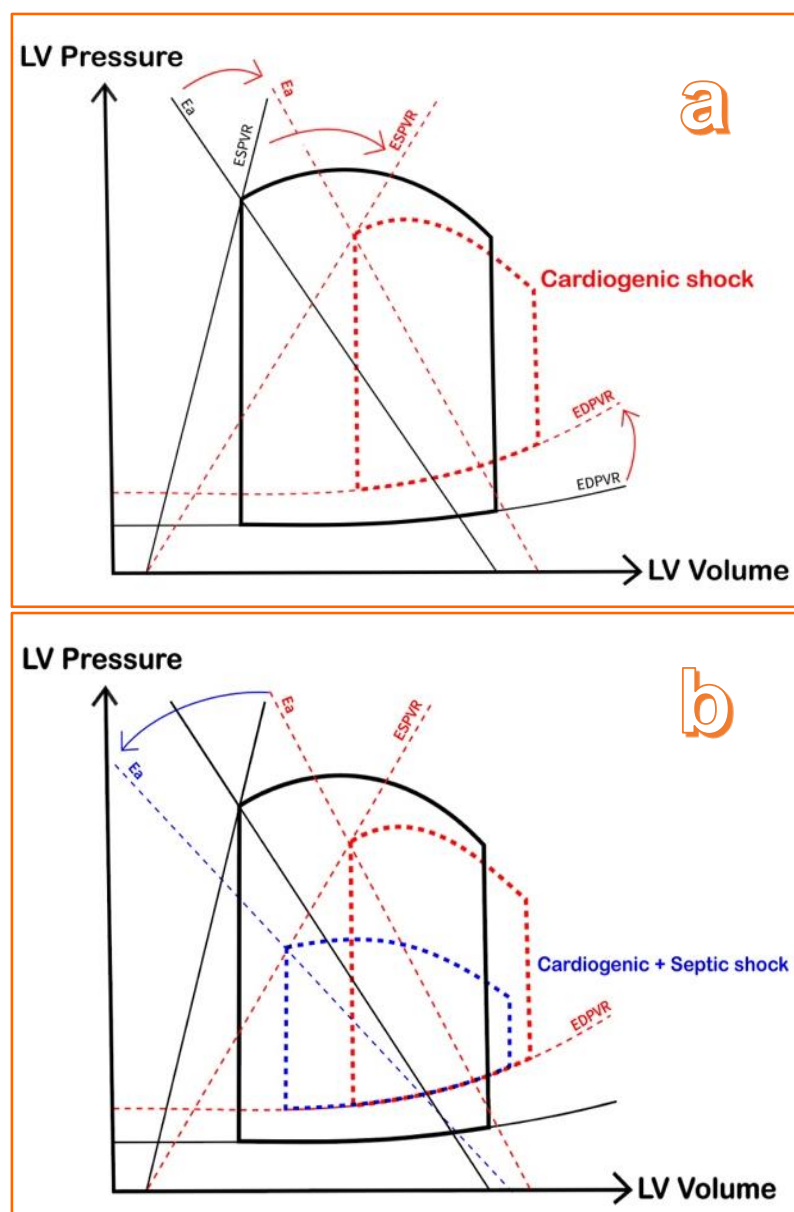

Figure 2: (a) Effects of cardiogenic shock on the pressure-volume loop with right shifting, elevated end-diastolic pressure-volume relationship, flattened end-systolic pressurevolume relationship, and elevated arterial elastance (red dashed line). (b) Cardiogenic shock with an additional septic component, with reduced arterial elastance (blue dashed line)

systolic impairment, activation of the renin-angiotensinaldosterone axis will occur, causing fluid retention and further increase in LVEDV. ${ }^{41}$ On ECHO, there will be a decrease in the measured $\mathrm{EF}$ and $\mathrm{SV} .{ }^{39}$ Impaired LV relaxation is evidenced by the reduction in Mitral e' or the presence of $\mathrm{L}$ waves. With the increase in LVEDV and impaired LV compliance, the measured LV filling pressure will increase; thus, both mitral E wave velocity and mitral E/e' ratio will increase. ${ }^{39}$ The calculated Ea will increase due to a significant decrease in SV and compensatory vasoconstriction. Ees derived from the single-beat method or by LVOT acceleration will decrease, resulting in ventriculo-arterial decoupling (Figure 2a). ${ }^{42}$ Interpretation of the ECHO findings in cardiogenic shock could be complicated, as impaired LV systolic function with diminished EF and SV can mask 
the effect of a sudden change in heart loading status. Sudden normalization of EF and SV may represent a marked decrease in afterload, causing a significant decrease in LVESV and an increase in SV (Figure 2b). ${ }^{43}$ In contrast, rapid normalization of $\mathrm{EF}$ with further worsening of SV in the presence of low LV filling pressure may indicate that the patient may have a decrease in preload status, which leads to the decline in LVESV and LVEDV with a further drop in cardiac output.

\subsection{Distributive Shock}

Distributive shock is characterized by alterations in tissue perfusion resulting from abnormal control of the microvasculature with abnormal distribution of a normal or increased cardiac output. ${ }^{44}$ Clinically, there is a significant decrease in $\mathrm{SVR},{ }^{45}$ which results in a depressed Ea curve on the PV loop. LV contractility will increase due to autonomic nervous system activation, and Ees will also increase. ${ }^{46}$ With the decrease in Ea and increase in Ees, SV and LVEF will increase, ${ }^{43}$ and LVESV will be significantly decreased. LV filling will decrease due to venodilatation and interstitial fluid loss, with a decrease in LVEDV. ${ }^{47}$ However, changes in LV filling pressure will depend on LV compliance. Measured LVESV and LVEDV will decrease with a significant increase in EF on ECHO. LV will appear hyperdynamic and well-filled. ${ }^{48}$ The measured SV will increase despite a collapsed $\mathrm{LV},{ }^{49}$ and the measured $\mathrm{LV}$ filling pressure will decrease due to decreased LV filling. ${ }^{50}$ Due to the decrease in systolic blood pressure and increased stroke volume, the calculated Ea would decrease substantially. Appropriate compensation in contractility will result in an increase in measured Ees (Figure 3a), while the failure to increase contractility will result in ventriculo-arterial decoupling. ${ }^{24}$

Septic shock is characterized by a hyperdynamic cardiovascular status with marked vasodilatation. However, various factors can alter patients' cardiovascular status and complicate the interpretation. Peripheral vasodilatation during sepsis can mask coexistent cardiac dysfunction. ${ }^{50}$ Vieillard-Baron et al. ${ }^{49}$ ] demonstrated that $35 \%$ of the patients with septic shock had a hypokinetic state at admission, as defined by a low cardiac index $\left(<3 \mathrm{~L} / \mathrm{min} / \mathrm{m}^{2}\right)$. In the case of septic shock, the incidence of sepsis-induced cardiomyopathy ranges from $10-70 \% .{ }^{51}$ These patients may present with paradoxically normal or low-normal EF and SV as a result of concomitant impaired contractility. The measured Ees should be normal or should decrease, and the calculated Ea should increase, resulting in ventriculo-arterial decoupling, ${ }^{24}$ (Figure 3b). Infusion of noradrenaline has been shown to increase Ea and MAP in patients with septic shock, while cardiac output would only be increased in patients with high Ees and normalized VA VA

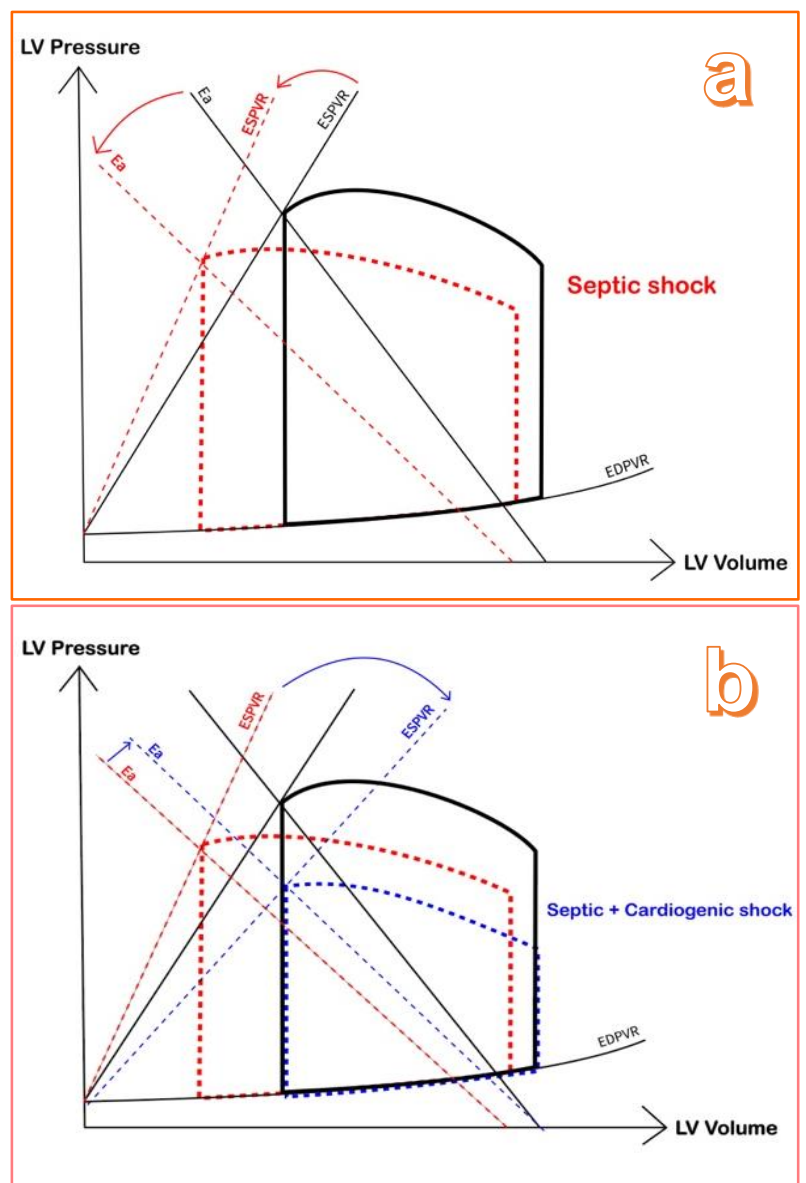

Figure 3: (a) Effects of distributive or septic shock on the pressure-volume loop, with left shifting, flatter arterial elastance, and compensatory elevated end-systolic pressurevolume relationship (red dashed line).

(b) Change in the pressure-volume loop with impaired left ventricular contractility in addition to distributive shock: elevated arterial elastance and flatter end-systolic pressure-volume relationship (blue dashed line).

VA coupling. ${ }^{51}$ In patients with low pre-infusion Ees and patients with excessive doses of noradrenaline, vasopressor administration can markedly decrease cardiac output and increase Ea, resulting in ventriculoarterial uncoupling. ${ }^{51}$

\subsection{Hypovolemic Shock}

The acute loss of intravascular volume due to severe bleeding or major fluid shift will decrease the mean systemic pressure and thus diminish venous return to the heart. The resultant decrease in LV preload will decrease LV output and lead to hypotension, leading to hypovolemic shock. On the PV loop, there will be a marked decrease in SV, LVEDV and LVEDP; and LVEF will increase. ${ }^{43}$ On ECHO, we can see that the LV is 
small and even collapsed with a "kissing wall". ${ }^{48}$ The measured LV filling pressure will decrease as the preload decreases. To compensate for decreased LV output, both LV contractility and arterial vascular tone will increase to restore blood pressure, ${ }^{52}$ so calculated Ea and measured Ees will increase with the appropriately responding LV (Figure 4a). If the patient's heart or vasculature fails to compensate for the loss in preload, there will be a significant decrease in blood pressure (Figure 4b). ${ }^{53}$ Hypovolemic patients with reduced preload tend to have positive fluid responsiveness; thus, volume expansion with fluid challenge will improve both arterial blood pressure and cardiac output, resulting in an increase in Ees and a decrease in Ea. ${ }^{51}$ However, positive fluid responsiveness is not necessarily equal to hypovolemia, ${ }^{54}$ while negative fluid responsiveness does not necessarily rule out hypovolemia. ${ }^{55}$ The topic of fluid responsiveness is out of the scope of discussion in this review. Table 1 summarizes the echo findings of the major subtypes of shock, and Figure 5 shows the results of potential interactions between them.

\section{Limitations}

The approach proposed above represents a methodical incorporation of various echocardiographic parameters to construct an individualized PV loop for each patient at a particular time point. The aim is to guide personalized treatment strategies tailored to the clinical situation. However, there are some limitations related to its clinical application. In daily practice, patients have always been given various kinds of treatments, and each of them have an effect on the contractility or loading status on the PV loop. The treatments may sometimes be given inappropriately or excessively, causing an adverse effect on patient hemodynamic conditions. Furthermore, the patient's own stress response could also affect the contractility and loading status, causing misinterpretation of ECHO. Therefore, we must consider the treatments being given and ensure that patients are not under stress and are adequately sedated to minimize the effect of stress on PV loop interpretation.

Patients' baseline cardiac function could also affect the interpretation of ECHO during shock. Patients with dilated cardiomyopathy have a dilated $\mathrm{LV}$ with reduced LVEF; thus, an ECHO finding of normal or slightly diminished EF with a mild increase in SV during shock could represent a significant distributive component. Patients with hypertrophic cardiomyopathy tend to have a higher LV filling pressure; thus, in the case of shock with ECHO, normal LV filling pressure may actually represent low LV filling pressure in patients where the diagnosis could be confirmed by testing for fluid responsiveness. Any cause of precapillary pulmonary hypertension with right ventricular failure can also affect the interpretation of the LV PV loop, as the decrease in

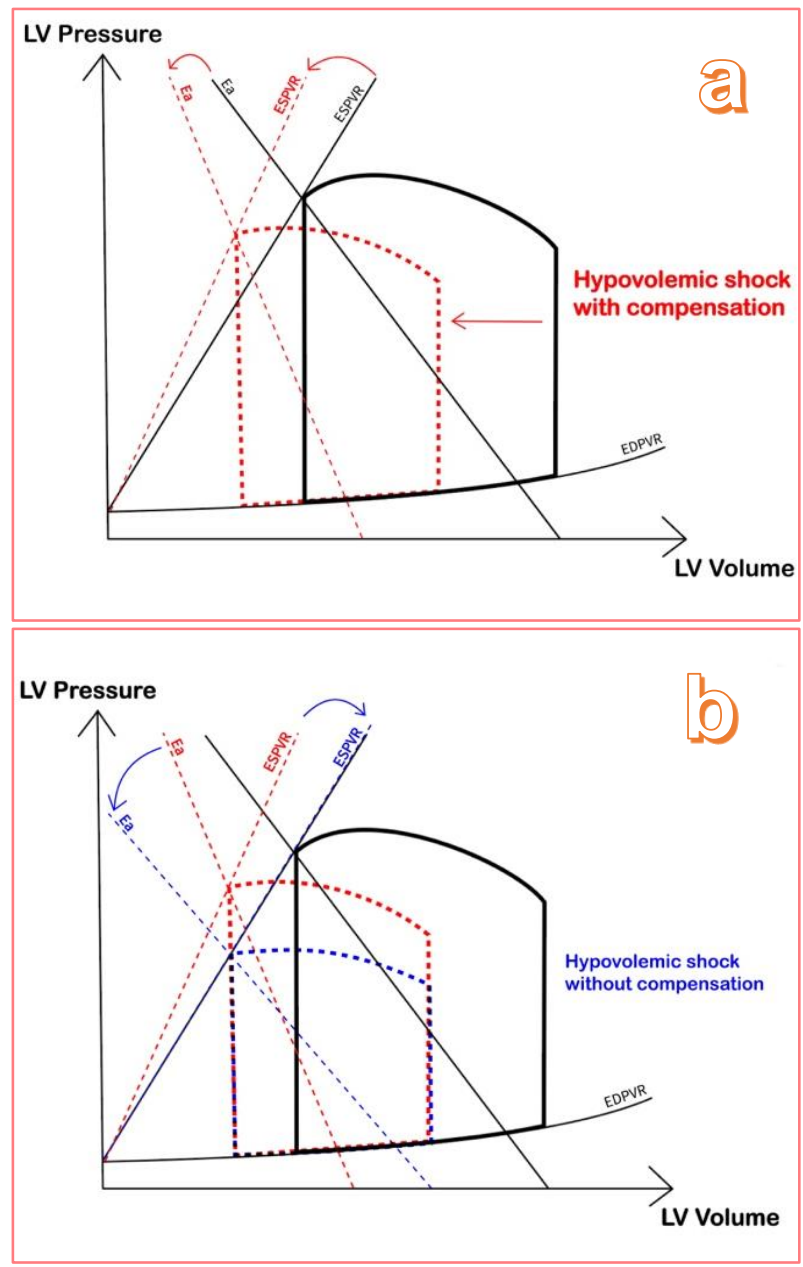

Figure 4: (a) Effects of compensated hypovolemic shock on the pressure-volume loop, causing left shifting, increased endsystolic pressure-volume relationship, and steeper arterial elastance (red dashed line).

(b) Pressure-volume loop in uncompensated hypovolemic shock, with reduction in arterial elastance and end-systolic pressure-volume relationship (blue dashed line).

RV output will mimic the hypovolemic state of the LV on ECHO, while patients may not be responsive to further fluid challenge because of interventricular interdependency. Therefore, patients' baseline cardiac function should be taken into account during the interpretation of ECHO findings.

The assessment of LV filling pressure allows for the correct estimation of LV preload and is also crucial for reflecting LV compliance in facing further fluid challenge. According to the 2016 American Society of Echocardiography Guidelines, LAP is determined by peak $\mathrm{E}$ velocity, mitral $\mathrm{E} / \mathrm{A}$ ratio, and additional parameters, such as average E/e' ratio, TR jet velocity, 
Table 1: Echocardiograph findings in different subtypes of shock.

\begin{tabular}{llll} 
Finding & Cardiogenic & Distributive & Hypovolemic \\
\hline LVEF & $\downarrow \downarrow$ & $\uparrow \uparrow \uparrow$ & $\uparrow \uparrow$ \\
\hline SV & $\downarrow \downarrow \downarrow$ & $\uparrow \uparrow$ & $\downarrow \downarrow$ \\
\hline $\begin{array}{l}\text { LV filling } \\
\text { pressure }\end{array}$ & $\uparrow$ & Normal/ $\downarrow$ & $\downarrow \downarrow \downarrow$ \\
\hline Ea & $\uparrow \uparrow \uparrow$ & & \\
\hline Ees & $\downarrow \downarrow$ & $\downarrow \downarrow \downarrow$ & $\uparrow$ \\
\hline $\begin{array}{l}\text { VA } \\
\text { coupling }\end{array}$ & $\uparrow \uparrow$ & $\uparrow \uparrow$ & $\uparrow$ \\
\hline
\end{tabular}

and LA maximum volume index..$^{15}$ However, those parameters are subject to changes in various cardiac conditions, and the resultant value may not truly reflect the existing LV filling pressure. TDI is a measurement of the velocity of tissue movement; thus, it is subject to change when there is a regional wall motion abnormality (e.g., hypertrophic cardiomyopathy, myocardial infarction, left bundle branch block, interventricular interdependency, annular calcification, or valve implant). TDI measurement, and hence those parameters derived from it, are also age-dependent and higher in the elderly. ${ }^{15}$ Beat-to-beat measurements of mitral flow and tissue Doppler parameters are subjected to changes during atrial fibrillation. TR jet velocity reflects pulmonary artery systolic pressure, which is a function of RV contractility and pulmonary vascular resistance. Therefore, in the case of increased pulmonary vascular resistance, the increased TR jet velocity will not truly reflect LA pressure. ${ }^{56}$ Various diseases will cause an increase in LA volume without an increase in LA pressure (e.g. atrial fibrillation). Additionally, acute changes in LA pressure will not cause an increase in LA volumes. ${ }^{57}$ When we encounter abnormal findings during LA pressure assessment, we need to correlate these findings with patients' clinical conditions. Various diastolic function assessment tools (e.g., pulmonary vein flow pattern and Valsalva maneuver) can help us to determine whether the patient has increased LA pressure. ${ }^{15}$

\section{Conclusion}

Bedside determination of cardiac contractility and loading status with transthoracic echocardiography allows physicians to understand the pathogenesis of shock and the interaction between different pathologies, and to predict the response to the various treatments given. Advanced transthoracic echocardiography training focusing on hemodynamic measurements is essential for acquiring accurate data for shock interpretation. Further studies on resuscitation targets based on patients' cardiovascular variables in the critical care setting are the next step toward an era of personalized resuscitation.

\section{Conflict of interests}

The authors declare that they have no competing interests.

\section{Authors' contributions}

TKB: Designed the concept, literature Search, drafted and revised this review

LCW, LKC: Literature Search, drafted and revised this review.

All authors read and approved the final manuscript

\section{References}

1. Cecconi M, Backer DD, Antonelli M, Beale R, Bakker J, Hofer $\mathrm{C}$, et al. Consensus on circulatory shock and hemodynamic monitoring. Task force of the European society of intensive care medicine. Intensive Care Med. 2014;40(12):1795-1815. [PubMed] DOI:10.1007/s00134-014-3525-z

2. Gershengorn HB, Wunsch $H$. Understanding changes in established practice: pulmonary artery catheter use in critically ill patients. Crit Care Med. 2013;41(12):2667-76. [PubMed] DOI: 10.1097/CCM.0b013e318298a41e

3. Harvey S, Harrison DA, Singer M, Ashcroft J, Jones CM, Elbourne D, et al. Assessment of the clinical effectiveness of pulmonary artery catheters in management of patients in intensive care (PAC-Man): a randomised controlled trial. Lancet. 2005;366(9484):472-7. [PubMed] DOI: $10.1016 / \mathrm{S} 0140-6736(05) 67061-4$

4. Wheeler AP, Bernard GR, Thompson BT, Schoenfeld D, Wiedemann HP, deBoisblanc B, et al. Pulmonary-artery versus central venous catheter to guide treatment of acute lung injury. N Engl J Med. 2006;354(21):2213-24. [PubMed] DOI: 10.1056/NEJMoa061895

5. Sandham JD, Hull RD, Brant RF, Knox L, Pineo GF, Doig CJ, et al. A randomized, controlled trial of the use of pulmonaryartery catheters in high-risk surgical patients. N Engl J Med. 2003;348(1):5-14. [PubMed] DOI: 10.1056/NEJMoa021108

6. Richard C, Warszawski J, Anguel N, Deye N, Combes A, Barnoud D, et al. Early use of the pulmonary artery catheter and outcomes in patients with shock and acute respiratory distress syndrome: a randomized controlled trial. JAMA. 2003;290(20):2713-20. [PubMed] DOI: 10.1001/jama.290.20.2713

7. Porter TR, S. Shillcutt SK, Adams MS, Desjardins G, Glas KE, Olson JJ, et al., Guidelines for the use of echocardiography as a monitor for therapeutic intervention in adults: a report from the American Society of Echocardiography. $\mathrm{J}$ Am Soc Echocardiogr. 2015;28(1):40-56. [PubMed] DOI: 10.1016/j.echo.2014.09.009.

8. Guyton AC, Hall JE. Textbook of Medical Physiology, Elsevier Saunders, Amsterdam, 2006. 
9. Pinsky MR, Payen D. Functional hemodynamic monitoring: foundations and future. Functional Hemodynamic Monitoring. 2005;42:3-6. DOI: 10.1007/3-540-26900-2_1

10. Kelly RP, Ting CT, Yang TM, Liu CP, Maughan WL, Chang MS, et al., Effective arterial elastance as index of arterial vascular load in humans. Circulation. 1992;86(2):513-21. [PubMed] DOI: 10.1161/01.cir.86.2.513

11. Redfield MM, Jacobsen SJ, Borlaug BA, Rodeheffer RJ, Kass DA. Age- and gender-related ventricular-vascular stiffening. Circulation. 2005;112(15):2254-62 [PubMed] DOI: 10.1161/CIRCULATIONAHA.105.541078

12. Chen $\mathrm{CH}$, Fetics $\mathrm{B}$, Nevo $\mathrm{E}$, Rochitte $\mathrm{CE}$, Chiou KR, Ding PA, et al. Noninvasive single-beat determination of left ventricular end-systolic elastance in humans. J Am Coll of Cardiol. 2001; 38(7):2028-34. [PubMed] DOI: 10.1016/s07351097(01)01651-5

13. Takeuchi M, Igarashi $\mathrm{Y}$, Tomimoto $\mathrm{S}$, Odake M, Hayashi T, Tsukamoto T, et al. Single-beat estimation of the slope of the end-systolic pressure-volume relation in the human left ventricle. Circulation. 1991;83(1):202-12. [PubMed] DOI: 10.1161/01.cir.83.1.202

14. Cope DK, Grimbert F, Downey JM, Taylor AE. Pulmonary capillary pressure: a review. Crit Care Med. 1992;20(7):104356. [PubMed] DOI: 10.1097/00003246-199207000-00024

15. Nagueh SF, Smiseth OA, Appleton CP, Byrd 3rd BF, Dokainish $\mathrm{H}$, Edvardsen $\mathrm{T}$, et al. Recommendations for the evaluation of left ventricular diastolic function by echocardiography: an update from the American society of echocardiography and the European association of cardiovascular imaging. J Am Soc Echocardiogr. 2016;29(4):277-314. [PubMed] DOI: 10.1016/j.echo.2016.01.011

16. Ishida Y, Meisner JS, Tsujioka K, Gallo JI, Yoran C, Frater RW, et al. Left ventricular filling dynamics: influence of left ventricular relaxation and left atrial pressure. Circulation. 1986;74(1):18796. [PubMed] DOI: 10.1161/01.cir.74.1.187

17. Nagueh SF, Middleton KJ, Kopelen HA, Zoghbi WA, Quiñones MA. Doppler tissue imaging: a noninvasive technique for evaluation of left ventricular relaxation and estimation of filling pressures. J Am Coll Cardiol. 1997;30(6):1527-33. [PubMed] DOI: 10.1016/s0735-1097(97)00344-6

18. Juhl-Olsen $P$, Hermansen JF, Frederiksen CA, Rasmussen LA, Jakobsen CJ, Sloth E. Positive end-expiratory pressure influences echocardiographic measures of diastolic function: a randomized, crossover study in cardiac surgery patients. Anesthesiology. 2013;199(5):1078-86. [PubMed] DOI: 10.1097/ALN.0b013e3182a10b40

19. Mullens W, Borowski AG, Curtin RJ, Thomas JD, Tang WH. Tissue Doppler imaging in the estimation of intracardiac filling pressure in decompensated patients with advanced systolic heart failure. Circulation. 2009;119(1):62-70. [PubMed] DOI: 10.1161/CIRCULATIONAHA.108.779223

20. Zile MR, Brutsaert DL. New concepts in diastolic dysfunction and diastolic heart failure: part II. Circulation. 2002;105(12):1503-8. [PubMed] DOI: 10.1161/hc1202.105290

21. Her AY, Kim JY, Choi EY, Kim SA, Jae RS, Shim CY, et al. Value of ventricular stiffness index and ventriculoarterial interaction in patients with nonischemic dilated cardiomyopathy. Circulation. 2009;73(9):1683-90. [PubMed] DOI: 10.1253/circj.cj-09-0046

22. Suga H. Time course of left ventricular pressure-volume relationship under various enddiastolic volume. Japanese Heart Journal.. 1969;10(6):509-15. [PubMed] DOI: 10.1536/ihj.10.509

23. Chantler PD, Lakatta EG, Najjar SS. Arterial-ventricular coupling: mechanistic insights into cardiovascular performance at rest and during exercise. Journal of Applied Physiology. 1985;105(4):1342-51. [PubMed] DOI: 10.1152/japplphysiol.90600.2008

24. Guarracino F, Ferro B, Morelli A, Bertini P, Baldassarri R, Pinsky MR. Ventriculoarterial decoupling in human septic shock. Crit Care. 2014;18(2):R80. [PubMed] DOI: 10.1186/cc13842

25. Prabhu SD. Altered left ventricular-arterial coupling precedes pump dysfunction in early heart failure. Heart Vessels. 2007;22(3):170-7. [PubMed] DOI: 10.1007/s00380-0060954-9

26. Sonnenblick EH. Force-velocity relations in mammalian heart muscle. Am J Physio. 1962;202:931. [PubMed] DOI: 10.1152/ajplegacy.1962.202.5.931

27. Weidemann F, Jamal F, Sutherland GR, Claus P, Kowalski M, Hatle $L$, et al. Myocardial function defined by strain rate and strain during alterations in inotropic states and heart rate. Am J Physiol Heart Circ Physiol. 2002;283(2):H792-9. [PubMed] DOI: 10.1152/ajpheart.00025.2002

28. Bennett ED, Barclay SA, Davis AL, Mannering D, Mehta N. Ascending aortic blood velocity and acceleration using Doppler ultrasound in the assessment of left ventricular function. Cardiovasc Res. 1984;18(10):632-8. [PubMed] DOI: 10.1093/cvr/18.10.632

29. Bauer F, Jones M, Shiota T, Firstenberg MS, Qin JX, Tsujino $\mathrm{H}$, et al. Left ventricular outflow tract mean systolic acceleration as a surrogate for the slope of the left ventricular end-systolic pressure-volume relationship. J Am Coll Cardiol. 2002;40(7):1320-7. [PubMed] DOI: 10.1016/s07351097(02)02138-1

30. Baumgartner $\mathrm{H}$, Hung J, Bermejo J, Chambers JB, Edvardsen $T$, Goldstein $S$, et al. Recommendations on the Echocardiographic Assessment of Aortic Valve Stenosis: A Focused Update from the European Association of Cardiovascular Imaging and the American Society of Echocardiography. Journal of the American Society of Echocardiography. 2017;30(4):373-92. [PubMed] DOI: 10.1016/j.echo.2017.02.009

31. Guzzetti E, Capoulade R, Tastet L, Garcia J, le Ven F, Arsenault M, et al. Estimation of Stroke Volume and Aortic Valve Area in Patients with Aortic Stenosis: A Comparison of Echocardiography versus Cardiovascular Magnetic Resonance. J Am Soc Echocardiogr. 2020;33(8):953-63. [PubMed] DOI: 10.1016/j.echo.2020.03.020

32. Singer M, Deutschman CS, Seymour CW, Shankar-Hari M, Annane D, Bauer M, et al. The Third International Consensus Definitions for Sepsis and Septic Shock (Sepsis-3). JAMA. 2016;315(8):801-10. [PubMed] DOI: 10.1001/jama.2016.0287 
33. Sunagawa K, Maughan WL, Burkhoff D, Sagawa K. Left ventricular interaction with arterial load studied in isolated canine ventricle. Am J Physiol. 1983;245(5):H773-H780. [PubMed] DOI: 10.1152/ajpheart.1983.245.5.H773

34. Blaudszun G, Morel DR. Relevance of the volume-axis intercept, $\mathrm{V}_{0}$, compared with the slope of end-systolic pressure-volume relationship in response to large variations in inotropy and afterload in rats. Exp Physiol. 2011;96(11):1179 95. [PubMed] DOI: 10.1113/expphysiol.2011.059881

35. Chen X, Liu F, Xu H, Zha D, Xiu J, Guo J, et al. Left ventricular diastolic dysfunction in patients with ST-elevation myocardial infarction following early and late reperfusion by coronary intervention. Int $\mathrm{J}$ Cardiol. 2017;228:886-89. [PubMed] DOI: 10.1016/j.ijcard.2016.11.146

36. Burri MV, Nanda NC, Lloyd SG, Hsiung MC, Dod HS, Beto RJ, et al. Assessment of Systolic and Diastolic Left Ventricular and Left Atrial Function Using Vector Velocity Imaging in Takotsubo Cardiomyopathy. Echocardiography. 2008;25(10):1138-44. [PubMed] DOI: 10.1111/j.1540-8175.2008.00819.x

37. Park S-M, Prasad A, Rihal C, Bell MR, Oh JK. Left Ventricular Systolic and Diastolic Function in Patients With Apical Ballooning Syndrome Compared With Patients With Acute Anterior ST-Segment Elevation Myocardial Infarction: A Functional Paradox. Mayo Clinic Proc. 2009;84(6):514-521. [PubMed] DOI: 10.4065/84.6.514

38. Bastos MB, Burkhoff D, Maly J, Daemen J, den Uil CA, Ameloot $\mathrm{K}$, et al. Invasive left ventricle pressure-volume analysis: overview and practical clinical implications. Eur Heart $\mathrm{J}$. 2020;41(12):1286-97.

DOI: 10.1093/eurheartj/ehz552

[PubMed]

39. Ky B, French B, May Khan A, Plappert T, Wang A, Chirinos JA, et al. Ventricular-Arterial Coupling, Remodeling, and Prognosis in Chronic Heart Failure. J Am Coll Cardiol. 2013;62(13):116572. [PubMed] DOI: 10.1016/j.jacc.2013.03.085

40. Tarazi RC, Levy MN. Cardiac responses to increased afterload. State-of-the-art review. Hypertension. 1982;4(3_pt_2): 8-18. [PubMed] DOI: 10.1161/01.HYP.4.3_Pt_2.8

41. Michael IB, Hannah RR, Daniel B. Pathophysiology and Advanced Hemodynamic Assessment of Cardiogenic Shock. Methodist Debakey Cardiovasc J. 2020;16(1):7-15. [PubMed] DOI: 10.14797/mdcj-16-1-7

42. Fox JM, Maurer MS. Ventriculovascular coupling in systolic and diastolic heart failure. Curr Heart Fail Resp. 2005;2(4):204-211. [PubMed] DOI: 10.1007/BF02696651

43. Monge García MI, Jian Z, Hatib F, Settels JJ, Cecconi M, Pinsky MR. Dynamic Arterial Elastance as a Ventriculo-Arterial Coupling Index: An Experimental Animal Study. Front Physiol. 2020;11:284. [PubMed] DOI: 10.3389/fphys.2020.00284

44. Vincent J-L. Hemodynamic support in septic shock. Intensive Care Med. 2001;27(14):S80-92. [PubMed] DOI: $10.1007 /$ PL00003799
45. Groeneveld ABJ, Nauta JJP, Thijs LG. Peripheral vascular resistance in septic shock: its relation to outcome. Intensive Care Med. 1988;14(2):141-47. [PubMed] DOI: $10.1007 / B F 00257468$

46. Monge García MI, Santos A. Understanding ventriculo-arterial coupling. Ann Transl Med. 2020;8(12):795. [PubMed] DOI: 10.21037/atm.2020.04.10

47. Rackow EC, Astiz ME. Mechanisms and Management of Septic Shock. Crit Care Clin. 1993;9(2):219-37. [PubMed] DOI: 10.1016/S0749-0704(18)30194-5

48. McLean AS. Echocardiography in shock management. Crit Care. 2016;20(1):275. [PubMed] DOI: 10.1186/s13054-016$1401-7$

49. Vieillard-Baron A, Prin S, Chergui K, Dubourg O, Jardin F. Hemodynamic Instability in Sepsis. Am J Respir Crit Care Med. 2003;168(11):1270-76. [PubMed] DOI: 10.1164/rccm.200306$816 \mathrm{CC}$

50. Vieillard-Baron A. Septic cardiomyopathy. Ann Intensive Care. 2011;1(1):6. [PubMed] DOI: 10.1186/2110-5820-1-6

51. Guarracino F, Bertini P, Pinsky MR. Cardiovascular determinants of resuscitation from sepsis and septic shock. Crit Care. 2019;23(1):118. [PubMed] DOI: 10.1186/s13054-0192414-9

52. Blomqvist CG, Stone HL. Cardiovascular Adjustments to Gravitational Stress. In: Comprehensive Physiology. Hoboken, NJ, USA: John Wiley \& Sons, Inc. 2011:][p1025-63.

53. Schiller AM, Howard JT, Convertino VA. The physiology of blood loss and shock: New insights from a human laboratory model of hemorrhage. Exp Biol Med (Maywood). 2017;242(8):874-83. DOI: $10.1177 / 1535370217694099$

[PubMed]

54. Takala J. Volume responsive, but does the patient need volume? Intensive Care Med. 2016;42(9):1461-63. [PubMed] DOI: 10.1007/s00134-015-4172-8

55. Sabatier C, Monge I, Maynar J, Ochagavia A. Assessment of cardiovascular preload and response to volume expansion. Med Intensiva. 2012;36(1):45-55. [PubMed] DOI: 0.1016/j.medin.2011.04.005

56. Vieillard-Baron A, Naeije R, Haddad F, Bogaard HJ, Bull TM, Fletcher $\mathrm{N}$, et al. Diagnostic workup, etiologies and management of acute right ventricle failure. Intensive Care Med. 2018;44(6):774-90. [PubMed] DOI: 10.1007/s00134018-5172-2

57. Bakkestrøm R, Andersen MJ, Ersbø\|l M, Bro-Jeppesen J, Gustafsson F, Køber L, et al. Early changes in left atrial volume after acute myocardial infarction. Relation to invasive hemodynamics at rest and during exercise. Int $\mathrm{J}$ Cardiol. 2016;223:717-22. [PubMed] DOI: 10.1016/j.jjcard.2016.08.228 